\title{
String universality in six
}

\author{
dimensions
}

\author{
Vijay Kumar and Washington Taylor
}

Center for Theoretical Physics, Massachusetts Institute of Technology, Cambridge, MA 02139, USA

vijayk@kitp.ucsb.edu; wati@mit.edu

\begin{abstract}
In six dimensions, cancellation of gauge, gravitational, and mixed anomalies strongly constrains the set of quantum field theories, which can be coupled consistently to gravity. We show that for some classes of six-dimensional (6D) supersymmetric gauge theories coupled to gravity, the anomaly cancellation conditions are equivalent to tadpole cancellation and other constraints on the matter content of heterotic/type I compactifications on K3. In these cases, all consistent $6 \mathrm{D}$ supergravity theories have a realization in string theory. We find one example that may arise from a novel string compactification, and we identify a new infinite family of models satisfying anomaly factorization. We find, however, that this infinite family of models, as well as other infinite families of models previously identified by Schwarz are pathological. We suggest that it may be feasible to demonstrate that there is a string theoretic realization of all consistent 6D supergravity theories which have Lagrangian descriptions with arbitrary gauge and matter content. We attempt to frame this hypothesis of string universality as a concrete conjecture.
\end{abstract}

e-print archive: http://lanl.arXiv.org/abs/0906.0987 


\section{Contents}

1 Introduction $\quad 326$

2 Examples of 6D models $\quad 328$

$2.1 \quad$ Gauge group $S U(N) \times S O(M) \quad 329$

2.2 Infinite families with factorized anomalies $\quad 335$

$\begin{array}{lll}2.3 & \text { On the existence of infinite families } & 337\end{array}$

3 String universality 339

$3.1 \mathcal{G}$ : Supersymmetric field theories with gravity in six dimensions $\quad 340$

$3.2 \mathcal{L}$ : The 6D SUSY string landscape 342

$3.3 \quad$ Statement of conjecture 342

3.4 Extending the conjecture $\quad 344$

$\begin{array}{lll}3.5 & \text { Predictivity } & 346\end{array}$

4 Conclusions $\quad 348$

$\begin{array}{ll}\text { Acknowledgment } & 349\end{array}$

$\begin{array}{ll}\text { References } & 349\end{array}$

\section{Introduction}

The vast plethora of apparently consistent string vacua has posed a challenge for string theory since the early days of the subject. Despite several decades of work, there is still no hint of any dynamical mechanism that might select one particular string vacuum solution. It seems clear at this point that there is an enormous range of distinct string compactifications giving rise to field theories in four dimensions through flux compactifications and related constructions [1]. In fact, it has been shown that there are discrete infinities of such string solutions [2]. Nonetheless, we are far from a full understanding of the range of possibilities, and there is an even larger set of low-energy field 
theories in four dimensions for which no obstruction is known to a consistent coupling to quantum gravity. The space of apparently consistent low-energy theories, which do not admit a realization in string theory was dubbed the "swampland" by Vafa [3]. In four dimensions, this swampland seems quite vast given the current state of our knowledge.

In six dimensions, the situation is quite different from that in four dimensions. The constraints posed by cancellation of gauge, gravitational, and mixed anomalies in dimensions $4 k+2$ are very stringent $[4,5]$. While the range of allowed gauge groups and massless field content in six dimensions is apparently quite large (see [6] for an extensive list and further references), the range of available string constructions through heterotic [7], F-theory [8-11], and other constructions is comparably extensive. The anomaly constraints essentially arise from an index theorem in the noncompact space-time dimensions, while the constraints on string constructions come from a combination of a tadpole constraint (related to an index in the compact space) and another application of the index theorem, describing the number of massless fields in a certain brane configuration or geometry. The anomaly constraints in six dimensions are closely related to the constraints on string compactifications [12-19].

In this paper, we consider some specific classes of six-dimensional (6D) field theories. We find that in these classes of models, the anomaly cancellation conditions in six dimensions are essentially equivalent to the tadpole and matter field constraints in string compactifications. In the previous literature [5], infinite families of models have been discovered, which satisfy anomaly factorization, a necessary condition for anomaly cancellation. No string compactification is known which corresponds to the theories in this infinite family. We find a new infinite class of theories, and show that these models, as well as those found in [5], are pathological. Motivated by the close relationship between the anomaly cancellation conditions in six dimensions and the constraints on string compactifications, and the relative paucity of examples of $6 \mathrm{D}$ supergravity theories, which are apparently consistent and yet lack a string description, we suggest that it may be within reach at the current time to give a definitive proof that string theory realizes all consistent, supersymmetric theories of quantum gravity in six dimensions. Furthermore, we expect that this class of consistent models can be determined entirely from the low-energy Lagrangian. We attempt to provide a framework for discussing and moving toward a proof of this hypothesis of string universality through a concrete conjecture.

In Section 2, we give examples of classes of models satisfying the anomaly cancellation conditions, and show that almost all of the models in these classes which appear to be consistent can be realized through existing string 
constructions. We discuss an apparent problem arising from the gauge kinetic terms of a large family of models which do not have string realizations. We also show that for the specific choice of gauge group $S U(N)$ with arbitrary matter content, there are only finitely many theories that can be consistently coupled to supergravity. In Section 3, we state the conjecture of string universality in six dimensions more precisely, and discuss various aspects of this hypothesis. Section 4 contains concluding remarks.

\section{Examples of $6 \mathrm{D}$ models}

A wide range of string constructions of supersymmetric $6 \mathrm{D}$ models have been studied in the literature. Some of the first models of this type were compactifications of type I or heterotic strings on K3 using line bundles [7]. More general vector bundle compactifications were described using F-theory in $[8,20,21]$. Compactifications on orbifolds give rise to a further range of theories [22,23], and gauge group enhancement from small instantons on singularities gives rise to a rich variety of $6 \mathrm{D}$ theories, which have been described in F-theory language [10].

Some work has also been done in characterizing 6D field theories, which exhibit cancellation of gauge, gravitational, and mixed anomalies, independent of string theoretic considerations [24-27]. In [5], several models (including two infinite families) were constructed which satisfy anomaly factorization. In [6], a systematic search for models satisfying anomaly cancellation was carried out, in the case where the gauge group has one or two factors, of which at most one factor is one of the classical groups $S U(N), S O(N)$, or $S p(N)$. Of the solutions of the anomaly cancellation conditions found so far, many correspond to known string constructions. Others do not.

In this section, we consider two classes of models. First, in Subsection 2.1, we look at models whose gauge group has the form $S U(N) \times S O(M)$, with certain types of allowed matter fields. We find that the anomaly cancellation conditions in this case are essentially equivalent to the conditions giving the tadpole constraint and matter content of a class of string compactifications, so that all of the models in this class which can be consistently coupled to gravity have a realization in string theory (with one possible exception). The same qualitative results hold with multiple factors of $S U(N)$ and a single $S O(M)$. We then consider in Subsection 2.2 several infinite families of models satisfying anomaly factorization, one of which arises in our analysis of the gauge group $S U(N) \times S O(M)$, the others of which were found in [5]. We find that these theories have wrong-sign kinetic terms at a general point 
in moduli space and seem to be pathological. Finally, we conclude this section with a demonstration in Subsection 2.3 that there are only finitely many anomaly-free models with gauge group $S U(N)$, with no restrictions whatsoever on allowed matter representations.

\section{$2.1 \quad$ Gauge group $S U(N) \times S O(M)$}

As an example of the correspondence between anomaly-free low-energy models and string constructions, we consider models with gauge group $S U(N) \times$ $S O(M)$. To simplify the analysis we restrict to $N \geq 4$ and $M \geq 4$. A similar analysis can be done for smaller values of $N, M$ with similar results, although the details are slightly more complicated.

The motivation for considering this particular class of models is that we know of a simple string construction giving one set of models of this type. These models were constructed through heterotic string compactifications using line bundles in [7], and studied further in [28]. In [29], we found a clean mathematical characterization of the general class of line bundle models constructed in [7] in terms of lattice embedding theorems of Nikulin. These results demonstrated that for a certain parameterization of possible models, all models could be constructed, in most cases in a unique fashion, through K3 string compactifications. Showing that all models in some appropriate class which satisfy anomaly cancellation can be realized through the parameterization of [29] would demonstrate string universality for this subclass of models, and furthermore prove uniqueness for the string realization of these models, up to some discrete redundancies.

The models constructed in $[7,29]$ are given by turning on $\mathrm{U}(1)$ fluxes in the type I compactification on K3. The resulting low-energy supersymmetric $6 \mathrm{D}$ theories have rank 16 gauge groups of the form

$$
U\left(N_{1}\right) \times U\left(N_{2}\right) \times \cdots \times U\left(N_{k}\right) \times S O\left(32-2 \sum_{i} N_{i}\right),
$$

with matter fields in the bifundamental representation connecting various components of the gauge group and in the antisymmetric representations of the components $U\left(N_{i}\right)$. The abelian $U(1)$ factors in the groups $U\left(N_{i}\right)$ are anomalous and acquire masses by the Green-Schwarz mechanism [30-32]. As a result, the gauge group contains $S U\left(N_{i}\right)$ factors with fewer neutral hypermultiplets. 
We consider theories with the gauge group $S U(N) \times S O(M)$ with $B$ massless hypermultiplets in the bifundamental representation $(N, M)+$ $(\bar{N}, M)$ and $A$ massless hypermultiplets in the two-index antisymmetric representation of $S U(N), \square+$ c.c ${ }^{1}$. In addition, these theories include the supergravity multiplet, one tensor multiplet and an arbitrary number of neutral hypermultiplets. The models we consider here are generalizations of the $k=1$ models in (1). We could also include hypermultiplets in the fundamental representations of these gauge groups; this would give a larger class of solutions including those realized by Higgsing the models under consideration here. For simplicity of the analysis, however, we restrict attention to the bifundamental and antisymmetric representations found in the models of $[7,29]$. This analysis naturally complements that of [6], where products of two groups were considered with zero or one classical factor.

In the heterotic/type I line bundle construction of $[7,29]$, the parameters $N, M, A, B$ satisfy the following constraints:

$$
\begin{aligned}
2 N+M & =32, \\
N \tau & =24, \\
A & =-2+4 \tau, \\
B & =-2+\tau,
\end{aligned}
$$

where $\tau$ is an integer parameter (in the notation of [29], $\tau=-f \cdot f / 2$ encodes the instanton number on a stack of $N$ D9-branes, and is integral because $f$ is a vector with even norm-squared in the homology lattice of a K3 surface). Conditions (2) and (3) arise from the D9-brane and D5-brane tadpole conditions, while (4) and (5) are computed from the index theorem on the D9-brane world volume giving the number of massless fields in each representation of the gauge group. These theories have 20 neutral hypermultiplets coming from the $\mathrm{K} 3$ moduli. One of these hypermultiplets is eaten by the (anomalous) $U(1)$ vector multiplet through the Green-Schwarz mechanism, leaving 19 neutral hypermultiplets. Our goal now is to show that (2)-(5) are equivalent to the anomaly cancellation conditions for the associated theories in six dimensions.

The gravitational and gauge anomalies in $6 \mathrm{D}$ supersymmetric theories arise from chiral fermions in hyper and vector multiplets of the supersymmetry (SUSY) algebra (as well as from the gravitino and self-dual and anti-selfdual 2-form fields). Anomaly cancellation in 6D models is described in [7],

\footnotetext{
${ }^{1}$ In six dimensions, hypermultiplets consist of two complex scalars, and can only be charged in a real representation of the gauge group. Henceforth, the complex conjugate is implicit if the representation is complex and we will drop it in our notation.
} 
and analyzed further in [33], which gives a concise summary of the anomaly cancellation conditions. We restrict attention here to theories admitting a Lagrangian description, so that the number of tensor multiplets is $n_{\mathrm{T}}=1$. For such models, cancellation of the $R^{4}$ anomaly requires the relation

$$
n_{\mathrm{H}}-n_{\mathrm{V}}=244,
$$

where $n_{\mathrm{H}}$ and $n_{\mathrm{V}}$ are the number of hyper- and vector multiplets, respectively. Since some number of hypermultiplets can be in the trivial representation of the gauge group, this is not a priori a strong constraint on allowed models; we will use this to compute the number of hypermultiplets transforming as singlets in anomaly-allowed low-energy theories and compare with the expected number of 19 (20-1) neutral hypers, which come from the closed string sector in general string compactifications.

The cancellation of the remaining gravitational, gauge, and mixed anomalies requires that the anomaly polynomial $I$ expressing these anomalies can be written in factorized form as required by the Green-Schwarz mechanism. The anomaly polynomial for the models we consider here is proportional to $[5,33]$

$$
\begin{aligned}
I= & \left(\operatorname{tr} R^{2}\right)^{2}+\frac{1}{6} \operatorname{tr} R^{2}\left(\operatorname{Tr} F_{2}^{2}-B N \operatorname{tr} F_{2}^{2}\right)+\frac{1}{6} \operatorname{tr} R^{2}\left(\operatorname{Tr} F_{1}^{2}\right. \\
& \left.-A \operatorname{tr}_{a} F_{1}^{2}-B M \operatorname{tr} F_{1}^{2}\right)-\frac{2}{3}\left(\operatorname{Tr} F_{2}^{4}-B N \operatorname{tr} F_{2}^{4}\right) \\
& -\frac{2}{3}\left(\operatorname{Tr} F_{1}^{4}-A \operatorname{tr}_{a} F_{1}^{4}-B M \operatorname{tr} F_{1}^{4}\right)+4 B \operatorname{tr} F_{1}^{2} \operatorname{tr} F_{2}^{2}
\end{aligned}
$$

where $\operatorname{Tr}$ denotes the trace in the adjoint representation, tr denotes the trace in the fundamental representation, and $\operatorname{tr}_{a}$ denotes the trace in the antisymmetric representation of $S U(N)$.

Using the trace identities [33]

$$
\begin{array}{ll}
S U(N): \quad & \operatorname{Tr} F^{2}=2 N \operatorname{tr} F^{2}, \\
& \operatorname{tr}_{a} F^{2}=(N-2) \operatorname{tr} F^{2}, \\
& \operatorname{Tr} F^{4}=2 N \operatorname{tr} F^{4}+6\left(\operatorname{tr} F^{2}\right)^{2}, \\
& \operatorname{tr}_{a} F^{4}=(N-8) \operatorname{tr} F^{4}+3\left(\operatorname{tr} F^{2}\right)^{2} . \\
S O(N): & \operatorname{Tr} F^{2}=(N-2) \operatorname{tr} F^{2}, \\
& \operatorname{Tr} F^{4}=(N-8) \operatorname{tr} F^{4}+3\left(\operatorname{tr} F^{2}\right)^{2}
\end{array}
$$


the polynomial simplifies to

$$
\begin{aligned}
I= & X^{2}+\frac{1}{6}(M-2-B N) X Z+\frac{1}{6}(2 N-A(N-2)-B M) X Y \\
& -2 Z^{2}+(2 A-4) Y^{2}+4 B Y Z \\
& -\frac{2}{3}(M-8-B N) \operatorname{tr} F_{2}^{4}-\frac{2}{3}(2 N-A(N-8)-B M) \operatorname{tr} F_{1}^{4},
\end{aligned}
$$

where $X:=\operatorname{tr} R^{2}, Y:=\operatorname{tr} F_{1}^{2}, Z:=\operatorname{tr} F_{2}^{2}$. We assume that $N \geq 4$ and $M \geq$ 4. Both $\operatorname{tr} F^{4}$ terms are then present for all relevant values of $N, M, A, B$. For anomaly cancellation, the polynomial must factorize, which requires any $\operatorname{tr} F^{4}$ terms to be absent. This gives two equations

$$
\begin{aligned}
M-B N & =8, \\
2 N-A N+8 A-B M & =0 .
\end{aligned}
$$

Substituting these conditions in $I$, we have

$$
I=X^{2}+(2 A-4) Y^{2}-2 Z^{2}-A X Y+4 B Y Z+X Z .
$$

For anomaly cancellation, $I$ must have the factorized form

$$
I=\left(X-\alpha_{1} Y-\alpha_{2} Z\right)\left(X-\tilde{\alpha}_{1} Y-\tilde{\alpha}_{2} Z\right) .
$$

The polynomial (10) can be factorized as above only if the following system of equations has a solution.

$$
\begin{aligned}
\alpha_{1}+\tilde{\alpha}_{1} & =A, \quad \alpha_{1} \tilde{\alpha}_{1}=2 A-4, \\
\alpha_{2}+\tilde{\alpha}_{2} & =-1, \quad \alpha_{2} \tilde{\alpha}_{2}=-2, \\
\alpha_{1} \tilde{\alpha}_{2}+\alpha_{2} \tilde{\alpha}_{1} & =4 B .
\end{aligned}
$$

There are two solutions to the pair of equations (13), namely $\alpha_{2}=1$, $\tilde{\alpha}_{2}=-2$ and $\alpha_{2}=-2, \tilde{\alpha}_{2}=1$. Since the factorized form is symmetric, without loss of generality we take

$$
\alpha_{2}=1, \quad \tilde{\alpha}_{2}=-2 .
$$

There are also two solutions to the pair of equations (12). The possibilities are

Case 1: $\quad \alpha_{1}=2, \quad \tilde{\alpha}_{1}=A-2$,

Case 2: $\quad \alpha_{1}=A-2, \quad \tilde{\alpha}_{1}=2$. 
In all known 6D string constructions giving gauge groups with a $U(N)$ factor, the associated value of the parameter $\alpha$ in the anomaly factorization condition is $\alpha=2[33,34]$. We now discuss these two cases in turn.

Case 1: $\alpha_{1}=2, \quad \tilde{\alpha}_{1}=A-2$

In this case, (14) combined with (15) gives

$$
\alpha_{1} \tilde{\alpha}_{2}+\alpha_{2} \tilde{\alpha}_{1}=A-6=4 B .
$$

This equation is precisely the relation between the numbers of matter fields dictated by (4), (5)

$$
\begin{aligned}
& A=-2+4 \tau, \\
& B=-2+\tau,
\end{aligned}
$$

where we can replace $B$ with the integer parameter $\tau$ through $B=\tau-2$. Thus, given cancellation of $F^{4}$ terms, factorization of the anomaly is equivalent to the constraints on the matter fields in the 10D compactification. Returning to the $F^{4}$ anomaly cancellation conditions, replacing $A$ and $B$ with $\tau$ through (18) gives

$$
\begin{aligned}
M+2 N-\tau N-8 & =0, \\
2 N+(2-4 \tau) N-16+32 \tau+(2-\tau) M & =0 .
\end{aligned}
$$

Subtracting (20) from twice (19) gives

$$
\tau(M+2 N-32)=0 .
$$

Since $B \geq 0, \tau \geq 2,(21)$ is equivalent to the D9-brane tadpole cancellation condition (2). Imposing this condition on (19) gives

$$
\tau N=24,
$$

which is precisely the D5-brane tadpole cancellation condition (3). Thus, in Case 1, the anomaly cancellation condition is precisely equivalent to the tadpole cancellation and matter contact constraints in a class of string vacuum constructions.

Case 2: $\alpha_{1}=A-2, \quad \tilde{\alpha}_{1}=2$

In this case, analogous to (17) we have the relation $A+2 B=3$. The only solutions to this for nonnegative $A, B$ are $(A, B)=(3,0),(1,1)$. For $(A, B)=$ $(3,0)$ substituting in $(9)$ gives the solution

$$
(N, M, A, B)=(24,8,3,0) .
$$


Table 1: Models solving anomaly cancellation conditions for $N \geq 4, M \geq 4$

\begin{tabular}{ccccc}
\hline & Gauge group & Anti $U(N)$ & $(N, M)$ & Neutral hypers \\
\hline 1 & $S U(4) \times S O(24)$ & 22 & 4 & 19 \\
2 & $S U(6) \times S O(20)$ & 14 & 2 & 19 \\
3 & $S U(8) \times S O(16)$ & 10 & 1 & 19 \\
4 & $S U(12) \times S O(8)$ & 6 & 0 & 19 \\
5 & $S U(24) \times S O(8)$ & 3 & 0 & 19 \\
6 & $S U(N) \times S O(N+8)$ & 1 & 1 & 271 \\
\hline
\end{tabular}

For $(A, B)=(1,1)$, there is an infinite family of solutions

$$
(N, M, A, B)=(N, N+8,1,1) .
$$

The anomaly free theories are shown in table 1 . Some comments are relevant regarding this list of models. In order to satisfy (6), which is the condition for the absence of purely gravitational anomalies, we add gauge singlet hypermultiplets. The number of neutral hypermultiplets in each case is computed using the gravitational anomaly cancellation condition (6), and included in table 1. In all cases except the infinite family (6), the number of hypermultiplets is 19 , as expected from a string compactification on K3.

We have found models (1-4) that have the same forms as known compactifications on K3. Extending the analysis for smaller $N$ gives additional models at $N=3,2$, associated with the compactifications found in $[7,29]$. We are not aware of an explicit string construction that realizes theory (5), but it seems reasonable to assume that such a realization exists. The theory has 19 neutral hypermultiplets, typical of K3 compactifications. The unusual value of $\alpha_{1}=1$, which differs from the value $\alpha=2$ typical of string constructions with a $S U(N)$ gauge group component [33,34], may give some hint for a string construction of this model. It is tempting to associate the $S U(24)$ with 24 D5-branes, which saturate the tadpole on K3, but that theory has gauge group $S p(24) \times S O(32)$ [35], since D5-branes in the type I theory carry a symplectic group.

Thus, all but one of theories (1-5) have a string construction, and it seems plausible that a string realization will also be found for the last one (5). The class of theories (6), on the other hand, seems problematic. This corresponds to an infinite family of $6 \mathrm{~d}$ gauge theories with increasing rank. In a K3 compactification, there seems to be an upper bound on the rank of the gauge group [10], and therefore it is questionable whether such an infinite family could ever be realized as a string compactification. However, as we show in the next section, this theory and other infinite families 
Table 2: Infinite families of anomaly-free $6 \mathrm{~d}$ theories, where the anomaly polynomial factorizes as shown. In each case, the number of neutral hypermultiplets can be calculated from the gravitational anomaly condition $n_{\mathrm{H}}-n_{\mathrm{V}}=244 . X, Y, Z$ denote $\operatorname{tr} R^{2}, \operatorname{tr} F_{1}^{2}, \operatorname{tr} F_{2}^{2}$, respectively, where $F_{1}$ is the field strength of the first gauge group factor and $F_{2}$ that of the second.

\begin{tabular}{lcc}
\hline Gauge group & Matter content & Anomaly polynomial \\
\hline$S U(N) \times S U(N)$ & $2(\square, \square)$ & $(X-2 Y+2 Z)(X+2 Y-2 Z)$ \\
$S O(2 N+8) \times S p(N)$ & $(\square, \square)$ & $(X-Y+Z)(X+2 Y-2 Z)$ \\
$S U(N) \times S O(N+8)$ & $(\square, \square)+\square$ & $(X+Y-Z)(X-2 Y+2 Z)$ \\
\hline
\end{tabular}

that have been found by Schwarz have a common pathology, which likely renders these theories inconsistent.

We have thus found that for this class of models there is a close correspondence between the anomaly cancellation conditions in six dimensions and the conditions determining the allowed gauge group and matter content in a class of string compactifications. A similar analysis can be done for other classes of models. We expect that in many other situations the results will be similar. For example, one can go to " $k$-stack" models with gauge group $S U\left(N_{1}\right) \times \cdots \times S U\left(N_{k}\right) \times S O(M)$. In this case, the equations have a similar structure and give rise to similar constraints. In fact, the equations governing these models are virtually identical to the analysis above, the only differences being that for each component $S U\left(N_{i}\right)$ there are separate numbers of multiplets $A_{i}, B_{i}$ and separate coefficients $\alpha_{i}, \tilde{\alpha}_{i}$ in the factorization equation (11) for each $i$, as well as a number of hypermultiplets $\beta_{i j}, \hat{\beta}_{i j}$ in the $\left(N_{i}, \bar{N}_{j}\right),\left(N_{i}, N_{j}\right)$ representations. Choosing the solution associated with Case 1 in (16) for all $i$ reproduces the equations for line bundle models with $k$ stacks, although the connection with string compactifications becomes more complicated at $k=4$. Choosing other cases gives a small number of other models analogous to the $S U(24) \times S O(8)$ model discussed above. We leave further analysis of these $k$-stack generalizations of the $S U(N) \times S O(M)$ models for further work.

\subsection{Infinite families with factorized anomalies}

The class of models (6) in table 1 gives an apparent infinite family of solutions to the anomaly cancellation equations. In searching for $6 \mathrm{D}$ models satisfying anomaly cancellation, Schwarz discovered two other infinite families of models satisfying anomaly factorization [5]. These infinite families are shown in table 2 along with (6) from table 1. 
It was shown by Sagnotti [27] (see also [36]) that the coefficients $\alpha_{i}, \tilde{\alpha}_{i}$ in the anomaly polynomial are related by SUSY to the coefficients of the gauge field kinetic terms in the Lagrangian. For the anomaly polynomial

$$
\begin{aligned}
I & =X_{4} \tilde{X}_{4}, \\
X_{4} & =\operatorname{tr} R^{2}-\sum_{i} \alpha_{i} \operatorname{tr} F_{i}^{2}, \\
\tilde{X}_{4} & =\operatorname{tr} R^{2}-\sum_{i} \tilde{\alpha}_{i} \operatorname{tr} F_{i}^{2},
\end{aligned}
$$

the gauge field kinetic terms in Einstein frame are

$$
\mathcal{L}_{\text {gauge }} \propto-\sum_{i}\left(\alpha_{i} e^{-\phi / 2}+\tilde{\alpha}_{i} e^{\phi / 2}\right) \operatorname{tr} F_{i}^{\mu \nu} F_{\mu \nu}^{i} .
$$

Here $\phi$ is the scalar in the tensor multiplet.

In all three infinite families shown in table $2, \alpha_{1}=-\alpha_{2}, \tilde{\alpha}_{1}=-\tilde{\alpha}_{2}$. As a result, the gauge kinetic term of one of the gauge group factors generically has the wrong sign, except at the special value $\exp (\langle\phi\rangle)=-\alpha_{1} / \tilde{\alpha}_{1}=-\alpha_{2} /$ $\tilde{\alpha}_{2}$. This corresponds to the case when both kinetic terms vanish and the $6 \mathrm{D}$ gauge coupling is infinite. The wrong-sign kinetic terms for at least one gauge group factor seem to indicate an instability, which renders these models inconsistent. Thus, we assume that these models cannot be sensibly interpreted as quantum supergravity theories in $6 \mathrm{D}$ with Lagrangian descriptions. Even more subtle "wrong sign" terms in low-energy Lagrangians have been shown to lead to inconsistencies in the UV [37]. It is possible, however, that there may be some unconventional way of making sense of these theories. By analogy with "ghost condensation" [38], it is possible that there is a phase transition to a new, Lorentz-violating vacuum in these infinite families; we are, however, interested in theories that are Lorentz invariant. There is a point in the tensor multiplet moduli space where both kinetic terms vanish. It was shown in [39] by Seiberg and Witten that at such points, the low-energy dynamics is controlled by tensionless strings and the tensor multiplet that is sourced by them; it is possible that the infinite sequences of models are actually redundant descriptions of some tensionless string theories, although these models would still be unstable with small fluctuations of the dilaton. It is also possible that these infinite families can be realized as an infinite sequence of unstable string compactifications ${ }^{2}$.

Note that we are interested here in theories that are coupled to gravity with a finite Newton constant. Thus, in string theory these should be

\footnotetext{
${ }^{2}$ Thanks to John McGreevy for discussions on this point.
} 
described by true string compactifications. If we relax this constraint, there is a much greater freedom in the construction of arbitrary 6D quantum field theories using "local" string constructions in terms of gauge fields on D-branes without gravity. The infinite family of $S U(N) \times S U(N)$ theories constructed by Schwarz can be realized by $N$ D5-branes at a $\mathbb{Z}_{2}$ singularity $[13,40]$. While this is a perfectly consistent family of gauge theories, it cannot be coupled to gravity without pathologies.

The apparent inconsistency of the known infinite families of anomaly-free theories in 6D supplies further evidence in support of the hypothesis of string universality in six dimensions, which we discuss in more detail in Section 3.

\subsection{On the existence of infinite families}

In the previous subsection, it was demonstrated that in the class of theories with gauge group $S U(N) \times S O(M)$ with $A$ hypermultiplets in Anti $S U(N)$ and $B$ hypermultiplets in $(N, M)$, there are only a finite number of anomalyfree models, which seem consistent. Although some infinite families have been found which apparently satisfy anomaly cancellation, these families exhibit pathologies which seem to rule them out as consistent gravity theories. It is interesting to ask whether anomaly cancellation alone is a sufficient condition to impose a finite constraint on the rank of the gauge group appearing in any consistent supergravity theory in six dimensions. It would be hard to realize gauge groups of arbitrarily large rank in a string compactification. A schematic argument for this is the fact that gauge groups come from $\mathrm{D}$-branes whose $\mathrm{R}-\mathrm{R}$ charge must be neutralized by negative tension objects like orientifold planes. Since these have a fixed negative charge, the number of D-branes that can be packed into a compact space is bounded from above, and so is the rank of the gauge group. Note that this argument is not airtight. In four dimensions, for example, D-branes in models with several $\mathrm{R}-\mathrm{R}$ tadpoles can have negative charge with respect to one or more of these tadpoles, making the proof of finiteness of the number of available models a rather nontrivial challenge [41]. It seems, however, in six dimensions, that the range of possibilities is sufficiently small that it is highly unlikely that any family of string compactifications giving gauge groups of arbitrarily high rank is possible.

Let us consider as an example the gauge group $S U(N)$ with $x_{i}$ multiplets in the representation $R_{i}$ of the gauge group. We look for the existence of an infinite family of theories with increasing rank, without any restrictions on $x_{i}$ and $R_{i}$. Anomaly-free models with gauge group $S U(N)$ were also studied in [6], although with only limited possible matter representations. 
The absence of gravitational anomalies requires $n_{\mathrm{H}}-n_{\mathrm{V}}=244$. For the theories in this class

$$
\sum_{i} x_{i} \operatorname{dim} R_{i}-N^{2}=243
$$

If there exists an infinite family, it follows that

$$
\lim _{N \rightarrow \infty}\left(\sum_{i} x_{i} \operatorname{dim} R_{i}-N^{2}\right) \rightarrow \mathcal{O}(1) .
$$

This condition restricts the possible representations $R_{i}$ to be among the fundamental, two-index symmetric, two-index anti-symmetric and adjoint representations, since any other representation has a dimension of order $\mathcal{O}\left(N^{3}\right)$. In fact, the only possibilities for the matter hypermultiplets consistent with (28) are

(1) 1 Adjoint +244 neutral,

(2) 1 Symmetric +1 Anti-symmetric +243 neutral,

(3) $N$ Fundamental +243 neutral,

(4) 2 Anti-symmetric $+N$ Fundamental +243 neutral,

(5) 1 Symmetric $+(N-1) / 2$ Fundamental +243 neutral, $N$ odd

(6) 1 Anti-symmetric $+(N+1) / 2$ Fundamental +243 neutral, $N$ odd.

In cases 1 and 2, the anomaly polynomial is just $I=\left(\operatorname{tr} R^{2}\right)^{2}$ and the kinetic term is zero. We discard these families as the gauge fields in these theories have no dynamics. In the remaining cases, the $\operatorname{tr} F^{4}$ term is present and nonvanishing for all $N$, and hence all such theories are anomalous.

We have thus shown that there exist only finitely many anomaly-free supersymmetric gauge theories with gauge group $S U(N)$ coupled to gravity in six-dimensions. A partial list of these theories with restrictions on the matter content can be found in [6]. The absence of infinite families implies that there is a maximal rank beyond which low-energy gauge theories with gauge group $S U(N)$ cannot be consistently coupled to supergravity in six dimensions. Although we do not have explicit string theoretic realizations of all theories in this class ${ }^{3}$, the fact that the gauge group rank is bounded from above, provides additional evidence for string universality in six dimensions. It would be interesting to find a general proof of a bound on the rank of

\footnotetext{
${ }^{3}$ In particular, three and higher index representations of $S U(N)$ would be hard to realize in a perturbative string compactification.
} 


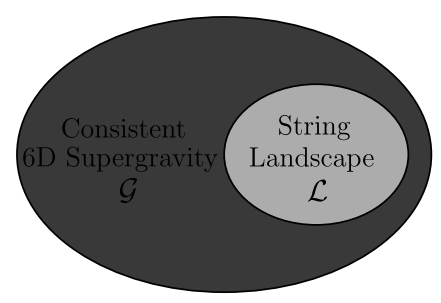

Figure 1: The set of consistent $6 \mathrm{D}$ gravity theories $\mathcal{G}$ contains the set of models realized through compactification of string theory to six dimensions $\mathcal{L}$. The conjecture of string universality states that $\mathcal{G}=\mathcal{L}$. If all models in $\mathcal{G}$ can be identified by features of the low-energy Lagrangian, then the swampland can be identified as $\mathcal{S}=\mathcal{G} / \mathcal{L}$, and in this situation string universality would imply that the $6 \mathrm{D}$ SUSY swampland is empty $(\mathcal{S}=\{\})$.

the gauge group for arbitrary forms of the gauge group in 6D supergravity theories purely based on anomaly cancellation arguments.

\section{String universality}

The close correspondence between the anomaly cancellation conditions in six dimensions and the conditions governing the tadpole constraints and matter field content of string compactifications suggests that the set of consistent 6D supersymmetric theories of matter coupled to gravity may be identified almost completely through anomaly cancellation conditions. Furthermore, this set of consistent models may be essentially the same as the set of supersymmetric $6 \mathrm{D}$ models which can be realized through string compactifications. In this section, we attempt to formulate a precise statement of how this correspondence may be realized. We denote the set of consistent $6 \mathrm{D}$ supergravity theories by $\mathcal{G}$, and the set of models that can be realized through some string compactification mechanism by $\mathcal{L}$. These sets of theories are defined more precisely in Subsections 3.1 and 3.2. Since all known compactifications of string theory to six dimensions give apparently consistent theories of quantum gravity, we assume $\mathcal{L} \subseteq \mathcal{G}$. The associated Venn diagram is depicted in figure 1 . The conjecture of string universality states that $\mathcal{G}=\mathcal{L}$. The conjecture is stated more precisely for the limited class of theories on which we focus in this paper in Subsection 3.3, where the related swampland picture of [3] is also discussed. The current situation regarding evidence for string universality in more general classes of theories, including dimensions other than six, is discussed in 3.4. Connections to the question of the predictivity of string theory are discussed briefly in 3.5 . 


\section{$3.1 \mathcal{G}$ : Supersymmetric field theories with gravity in six dimensions}

Supersymmetric gravity theories in six dimensions have a rich structure and have been widely studied in the literature $[24,25]$. Much of the richness of this structure arises in theories with multiple massless tensor multiplets $[23,26,27]$ and in limits with tensionless string excitations [39]. For simplicity, in this paper we focus on theories with a Lagrangian description, for which $n_{\mathrm{T}}=1$. Thus, we are interested in theories of $6 \mathrm{D}$ gravity with one $6 \mathrm{D}$ gravity SUSY multiplet, one tensor multiplet, and any number of vector- and hyper-multiplets $n_{\mathrm{V}}, n_{\mathrm{H}}$ associated with an arbitrary gauge group and matter content in any representations of the gauge group. While such theories are generally trivial in the infrared, we can study many of their properties from the supersymmetric Lagrangian description. In particular, from the structure of the gauge group and matter content we can analyze the anomaly structure of any given theory.

We define $\mathcal{G}$ to be the set of consistent $6 \mathrm{D}$ supersymmetric quantum theories of gravity with gauge symmetry and matter fields. This definition requires some discussion. We do not a priori have a systematic way of identifying which 6D Lagrangians are associated with consistent quantum theories of gravity. It is possible that string theory is the only way in which gravity can be consistently quantized in any dimension; some researchers in the field may take this as a working hypothesis, and in some sense the notion of string universality has been implicit in much work over the last several decades. It is, however, logically possible that string theory is a consistent quantization of some theories of gravity, but that there are other UV-complete quantum theories of gravity disjoint from the string theory landscape. Thus, suggesting that all consistent supergravity theories are described by string theory is not tautological, but is a hypothesis subject to mathematical proof or refutation. To prove such a hypothesis, a key step is finding criteria for determining which models lie in the set $\mathcal{G}$, independent of string theory. This means that we wish to find a set of conditions determining $\mathcal{G}$ which can be analyzed from the point of view of the low-energy Lagrangian. One of the main points of this paper is that in the case of $6 \mathrm{D}$ supersymmetric theories we may be very close to having a complete set of such conditions. As discussed above, anomaly cancellation gives a very strong set of conditions constraining the set of theories in $\mathcal{G}$. Some models that satisfy local anomaly cancellation, however, may still not admit a UV completion to a quantum theory of gravity. For example, global anomalies must also be taken into account [11]. For some models, extra massless fields may be required in order to satisfy unitarity. Or nontrivial topological states 
may be required for consistency of the theory. In this case, an additional condition for consistency of the quantum theory would involve the cancellation of anomalies on the world-volume of the topological excitation, as suggested in [18]. Other constraints on the low-energy Lagrangian required for consistency of supergravity theories in the UV are found in [37]. Thus, while we do not claim to have a complete set of criteria by which models in $\mathcal{G}$ can be determined, we suggest that at least for 6D supersymmetric theories, anomaly cancellation provides a strong litmus test, and that further development of consistency conditions based on low-energy considerations may lead to a complete determination of what models may admit consistent quantization independent of string theory considerations.

This discussion is closely related to the perspective presented by Vafa in [3], developed further in [42]. In that work, the "swampland" is defined to be the set of consistent-looking effective field theories, which are actually inconsistent. The implicit assumption in this work seems to be that any theory not realized in string theory is inconsistent, so that the swampland represents all theories which are consistent with what we know about the low-energy theory, but which cannot be realized in string theory. The extent of the swampland thus depends upon the power of our tools with which to probe the consistency of a theory from its low-energy description. In this context, the point of the present work is to suggest that in the case of $6 \mathrm{D}$ supergravity theories, we may be close to having a complete characterization of which low-energy theories are consistent. Instead of defining the space $\mathcal{G}$ of consistent gravity theories, we could have chosen to define a space $\mathcal{C}$ of "apparently consistent" theories of $6 \mathrm{D}$ gravity. The swampland would then be defined as the set $\mathcal{C} \backslash \mathcal{L}$. The difficulty with this definition is that it depends upon the set of tools we allow for testing consistency of a theory. If we restrict to anomaly cancellation, it would define a particular class of theories $\mathcal{C}$. There are, however, other criteria, including those mentioned above, which may be used to refine the set of apparently consistent theories based on the low-energy Lagrangian. The space $\mathcal{C}$ is therefore somewhat of a moving target, which will be reduced in scope as our tools for discovering quantum inconsistencies are improved, although we will always have $\mathcal{C} \supseteq \mathcal{G}$. For purposes of clarity, therefore, we have chosen to frame our discussion primarily in terms of the space $\mathcal{G}$, which represents a mathematically idealized static set, despite our current ignorance of how to determine this set. The goal is then to find a set of criteria, which are sufficiently stringent to define a set of "apparently consistent" models $\mathcal{C}$ which coincide with $\mathcal{G}$. Our discussion here is therefore similar in philosophy if not in semantics and notation, to the approach taken in $[3,42]$, where additional criteria based on finiteness of moduli space [43] and numbers of fields are suggested as ways in which the set $\mathcal{C}$ can be reduced. 


\section{$3.2 \mathcal{L}:$ The 6D SUSY string landscape}

There are many ways to construct $6 \mathrm{D}$ supersymmetric vacua in string theory. These include heterotic/type I compactifications on K3 [7], F-theory compactifications $[8,10,20,21,44]$, and orbifold constructions [22,23]. All these constructions are related through various duality symmetries (reviewed in [45]). It is quite possible that string constructions exist, which are related in some limits through duality to constructions which have already been studied, but which include new classes of vacua not yet considered. By $\mathcal{L}$ we mean the complete space of compactifications in the entire string theory duality web. Since string theory is not yet well-defined in a backgroundindependent fashion, we do not yet have a complete mathematical definition of $\mathcal{L}$. Nonetheless, if we assume that there is some as-yet unknown definition of string theory we can assume that there is some well defined, yet unknown definition of the set $\mathcal{L}$. Despite our ignorance about the general theory, we can at any time define a set $\mathcal{K}$ of known string theory constructions. Like the set $\mathcal{C}$ defined in the previous subsection, $\mathcal{K}$ is a moving target, which always satisfies $\mathcal{K} \subseteq \mathcal{L}$. The point we would like to emphasize here is that in six dimensions with SUSY, we may be close to understanding a wide enough range of string constructions that realizing $\mathcal{K}=\mathcal{L}$ may be within reach.

\subsection{Statement of conjecture}

The statement of string universality in the class of theories of interest in this paper can be stated as the following conjecture.

Conjecture. All $\mathcal{N}=(1,0)$ supersymmetric theories of gravity coupled to gauge fields and matter in six dimensions (with one gravity and one tensor multiplet) which are anomaly-free and consistent quantum theories can be realized through a compactification of string theory.

In terms of the sets of theories defined in the previous two subsections, this conjecture states that for a restricted class of $6 \mathrm{D}$ supergravity theories

$$
\mathcal{G}=\mathcal{L}
$$

The purpose in formulating this conjecture is to provide a concrete framework in which progress can be made in understanding the landscape of string vacua $(\mathcal{L})$, consistent quantum gravity theories $(\mathcal{G})$, and the relationship between these classes of theories. As discussed above, both $\mathcal{L}$ and $\mathcal{G}$ represent some mathematically idealized sets, which we do not yet know 
how to completely determine. We have tools for demonstrating that certain theories are inconsistent, leaving a set $\mathcal{C}$ of theories, as defined above, which are "apparently consistent". We have a set $\mathcal{K}$ of known string constructions. The relationship between these four sets is given by

$$
\mathcal{K} \subseteq \mathcal{L} \subseteq \mathcal{G} \subseteq \mathcal{C} .
$$

Even though we do not know precisely how to define the sets $\mathcal{L}$ and $\mathcal{G}$, we have definitions of $\mathcal{K}$ and $\mathcal{C}$, which over time form better bounds on the sets $\mathcal{L}$ and $\mathcal{G}$. One way to prove the conjecture that $\mathcal{L}=\mathcal{G}$ is to prove that $\mathcal{K}=\mathcal{C}$ since

$$
\mathcal{K}=\mathcal{C} \quad \Rightarrow \quad \mathcal{L}=\mathcal{G} .
$$

The point we would like to make here is that in $6 \mathrm{D}$ supergravity theories, our definitions of $\mathcal{K}$ and $\mathcal{C}$ are sufficiently good that we are close to having $\mathcal{K}=\mathcal{C}$, from which the stated conjecture would follow from (31).

As stated above, the purpose of making this conjecture is to provide a framework for refining our understanding of the string landscape and consistent gravity theories in an arena where short-term progress may be possible. In Section 2.1, we considered a simple class of 6D SUSY models with gauge groups $S U(N) \times S O(M)$. The analysis carried out in that subsection proves that for this class of models $\mathcal{C}=\mathcal{K}$ and therefore the conjecture of string universality holds in this limited class of models (with one uncertain exception which we expect also has a string realization and agrees with the conjecture). Here $\mathcal{C}$ can be defined as the set of supersymmetric, anomaly-free models, with the stated limitations on gauge group and matter content restricting to the subclass of theories of interest. We also considered in 2.2 several infinite families of models satisfying anomaly factorization, and showed that these models have wrong-sign gauge kinetic terms and therefore do not represent counterexamples to the conjecture.

There are several ways in which one might imagine making progress in proving the conjecture (29). The most straightforward is to consider models which do not have known string constructions, but which are not anomalous (i.e., which lie in $\mathcal{C} \backslash \mathcal{K}$ ). For any such model $m$ there are three possibilities:

(1) The model admits a string construction, so $m \in \mathcal{K}$ with an improved definition of $\mathcal{K}$.

(2) The model is inconsistent, so $m \notin \mathcal{C}$ for an improved $\mathcal{C}$.

(3) The model is a consistent theory of gravity but cannot be realized in string theory, so $m \in \mathcal{G} \backslash \mathcal{L}$, providing a counterexample to the conjecture of string universality. 
Thus, analyzing such models provides a systematic way of expanding/ refining the definitions of $\mathcal{K}, \mathcal{C}$. At present there are few models known of this type. Categorizing and analyzing these models may be helpful in increasing our understanding of the situation and approaching a demonstration of the conjecture stated above.

It is also possible that more abstract considerations may be useful in proving the conjecture (29), at least in restricted classes of theories. As shown in 2.1, in some cases the anomaly cancellation conditions in six dimensions are precisely equivalent to conditions on field theories derived from string compactifications. Proving this in a more general context, perhaps using the mathematics of index theories, might provide a deeper insight into the structure of the string landscape.

\subsection{Extending the conjecture}

We have stated the conjecture (29) in the restricted context of 6D supersymmetric theories with one tensor multiplet because it is in this arena that we have strong constraints on allowed theories of quantum gravity, which come close to defining the same set of models which can be realized using known string compactifications. One could imagine generalizing this conjecture in several directions.

For example, one could drop the restriction that $n_{\mathrm{T}}=1$. Unfortunately, our understanding of theories with more than one tensor multiplet in six dimensions is rather limited, outside the insights which have come from string theory (some of which are described in $[23,39]$ ). Thus, our understanding on the field theory side is rather limited. Nonetheless, anomaly cancellation conditions can still be determined based on the massless field content and representations of the theory $[26,27]$, so progress may be possible in this direction without the need for tremendous new insights.

The analogue of the conjecture made here in ten dimensions is a much simpler story, much of which is well known. Without the Green-Schwarz mechanism, anomaly cancellation in ten dimensions basically rules out all chiral supergravity theories other than the type IIB theory [4]. The GreenSchwarz mechanism [46] makes possible not only $\mathcal{N}=1$ supergravity coupled to the $\mathrm{SO}(32)$ gauge theory, but also the $E_{8} \times E_{8}$ theory (which led to the discovery of the corresponding string theory), and two additional theories with gauge group $U(1)^{496}$ and $E_{8} \times U(1)^{248}$. These latter theories have not been shown either to exhibit inconsistencies as quantum theories of gravity, or realized through string theory. (Thus, in the language of the previous subsection, these theories lie in the set $\mathcal{C} \backslash \mathcal{K}$.) The proof of 
the conjecture $\mathcal{G}=\mathcal{L}$ in ten dimensions would involve showing that these two theories either admit string constructions or are inconsistent. It seems most likely that these models are inconsistent ${ }^{4}$, as also suggested in $[3,47]$, although this remains to be proven. Discovering a mechanism for inconsistency of these theories may also be a useful step in proving the conjecture in six dimensions, where analogous anomaly-free models arise. In nine, and seven dimensions, the space of possible string compactifications is also much better understood [48] than in six dimensions. Thus, it may be interesting to attempt to prove string universality in these higher dimensions, which could give lessons relevant for more complicated lower-dimensional situations.

Clearly, the case of physical interest is that of $4 \mathrm{D}$ theories. One could conjecture that string universality holds in four dimensions, but this hypothesis is much less compelling than in six dimensions given our current state of knowledge, even if one restricts attention to the class of supersymmetric theories. For one thing, since there are no purely gravitational anomalies in four dimensions, the constraints on allowed field theories are much weaker. For another thing, our understanding of general string compactifications to four dimensions is much more limited than for six dimensions. Beyond the enormous number of relatively well-understood compactifications available through Calabi-Yau manifolds with branes and fluxes, F-theory compactifications on elliptically fibered Calabi-Yau 4-folds, and less well-understood M-theory compactifications on $G_{2}$ manifolds, there are also compactifications on non-Kähler manifolds, and perhaps also nongeometric manifolds, with which we are only beginning to come to grips. Thus, at this time in four dimensions, the definition of the set of known compactifications $\mathcal{K}$, while vast, is much smaller than the set of apparently consistent $4 \mathrm{D}$ theories coupled to gravity $\mathcal{C}$. This is the framework in which the discussion and analysis of the swampland in $[3,42]$ is most relevant. We need to make a vast improvement in our understanding of both the space of string compactifications and the set of criteria for showing that theories of gravity are quantum inconsistent in four dimensions to even begin to approach a situation where $\mathcal{C}=\mathcal{K}$. It may be that aspects of the finiteness criteria and moduli space structure discussed in $[3,42,43]$ play a very useful role in refining our understanding of these questions in four dimensions. It may be that some analogue of anomaly cancellation persists in four dimensions which can dramatically cut down on the number of apparently consistent gravity theories. It may be that string universality holds for 4D theories with SUSY, but that SUSY breaking mechanisms lead to a constrained subset of nonsupersymmetric low-energy theories in $4 \mathrm{D}$. Moreover, it may be that in four

\footnotetext{
${ }^{4}$ Thanks to Allan Adams and Joe Polchinski for discussions regarding these models.
} 
dimensions, even for supersymmetric theories, $\mathcal{G} \neq \mathcal{L}$. In any case, further study of all these questions is clearly of great value, but we are far from the point at which there is significant evidence for string universality in four dimensions.

\subsection{Predictivity}

At the heart of the current controversy about the string landscape is concern about the predictivity of the theory. If some dynamical principle would pick out a unique string vacuum, corresponding to our observed standard model of particle physics with some specific high-energy behavior and UV completion, then the string theory would be completely predictive and determining this vacuum would in principle allow us to determine precisely what physics to expect above the $\mathrm{TeV}$ scale. If, on the other hand, eternal inflation populates the landscape of theories, so that each string solution is realized in some regions of a vast "metaverse" [1,49], then if the solutions of string theory are sufficiently dense in the space of allowed low-energy field theories, predictions for physics well below the Planck or compactification scale would be difficult or impossible. It is possible that the dynamics of string cosmology may define a natural measure on the space of string solutions, which would favor some solutions over others. Currently, however, we lack a mathematically complete or background-independent formulation of string theory. It is likely that significant progress in this direction will be needed to understand the cosmological measure on the string landscape. In this brief discussion, we describe the situation for predictivity in the absence of such a breakthrough.

If it is correct, or even close to correct, that string universality holds in six dimensions, then in this case we seem in some sense to be in the worst possible situation vis a vis low-energy predictions. If every possible consistent theory can be identified from low-energy considerations, and all of these theories can be realized in string theory, then string theory would seem to have no predictive power for low-energy physics without some deep new insight into the structure of the theory. It has been suggested [50] that statistical methods may be useful in analyzing the distribution of string vacua, so that even with a large number of vacua densely populating the space of low-energy field theories, some specific values for parameters in the low-energy theory may be overwhelmingly favored by the vacuum statistics. On the other hand, if the special class of examples considered in [29] is typical, the situation may be that each possible consistent low-energy theory is realized in a single way in string theory, which may be essentially unique up to duality symmetries. 
If we were living in six dimensions, then this would seem like a very awkward situation for string theory. It should be emphasized, however, that there is no reason a priori why a theory of quantum gravity relevant at the Planck scale of $10^{19} \mathrm{GeV}$ should make any prediction for physics at the scale of $1 \mathrm{TeV}, 16$ orders of magnitude below the quantum gravity scale. String theory is valuable as a framework for describing quantum gravity. If in fact, string theory can be used to provide a UV completion of essentially any low-energy theory whose coupling to quantum gravity does not violate some basic principle like unitary via anomalies, this can be seen as a strength of the theory. There is a certain symmetry and elegance about the notion of a quantum gravity theory, which provides for the production of essentially all possible low-energy behaviors in some regime of the theory or region of the metaverse.

If indeed, string theory can give rise to such a wide range of low-energy behavior that predictions at the $\mathrm{TeV}$ scale cannot be made precisely, it may bother some scientists that this makes the theory difficult to test. However, on the other hand, this does not make the theory any less likely to be correct. It just makes it more difficult to verify. In fact, one interesting feature of the $6 \mathrm{D}$ theories we have considered in this paper is that they all contain a massless antisymmetric tensor field $B_{\mu \nu}$. These theories therefore contain a class of topological string-like defects which are charged under the $B$ field. It may well be that unitarity of these theories requires that such topological string excitations be included in the theory as quantum degrees of freedom. Because these string excitations are essentially equivalent to the fundamental string, the proof of string universality in six dimensions, along with a demonstration of the necessity for dynamical strings in these theories might show that not only does string theory represent a valid UV completion of each theory in this class, but that this UV completion would essentially be unique, and that all quantum gravity theories in this class would by necessity be string theories. Such an argument cannot work in four dimensions, where not all supersymmetric theories have an antisymmetric 2-form field, but some more general argument along these lines, including the possibility of M-theory compactifications and other more exotic arrangements, may be plausible.

In any case, these rather philosophical speculations apply in the case of six dimensions, where there is some evidence that the string theory gives rise to at least a good fraction of the consistent quantum gravity theories with SUSY. Where we live, in four dimensions with no manifest SUSY, our understanding of the constraints on allowed field theories and string constructions is still so weak that there is room for a large gap between the space of string constructions and viable low-energy field theories, which 
would give predictions for low-energy physics. In attempting to understand this problem better in six dimensions, where we are closer to a complete story, we will inevitably develop better tools for understanding the analogous situation in four dimensions. Even if, in the end, string theory does not provide a strong constraint on which apparently consistent low-energy field theories with gravity and SUSY admit a UV completion, probing these questions may give us more powerful tools for understanding which lowenergy theories admit a consistent coupling to gravity based on completely low-energy considerations; such tools may themselves provide new and interesting insights or predictions for low-energy physics. It is also possible that attaining a reasonably complete description of the range of allowed string vacua in four dimensions (i.e., approaching $\mathcal{K} \rightarrow \mathcal{L}$ ) may be a goal which is attained more readily than developing tools for determining the inconsistency of quantum gravity theories in four dimensions (i.e., approaching $\mathcal{C} \rightarrow \mathcal{G}$ ). In this case, string theory can provide a key to understanding how observable physics may be consistently extended to higher energies. In any case, the notion that string theory provides a more or less complete catalogue of all possible low-energy theories compatible with quantum gravity, even in six dimensions, suggests that any new and unexpected phenomena found in experiments at higher energies may be realizable in the string theory context, so that, as has been the case for many years, string theory can provide a valuable tool for understanding the range of possible mechanisms for low-energy phenomena, such as SUSY, supersymmetric breaking mechanisms, unification, flavor symmetry, and perhaps any new physics at the $\mathrm{TeV}$ scale which will be discovered in coming years.

\section{Conclusions}

In this paper, we have shown that cancellation of gravitational, gauge, and mixed anomalies gives a sufficient constraint on 6D supersymmetric theories of gravity with gauge and matter fields that in some cases all models consistent with anomaly cancellation admit a realization through string theory. We have ruled out a number of infinite families of models, which satisfy anomaly factorization, so that the gap is rather small between the set of known 6D models satisfying anomaly cancellation and the set of models realized through string compactification. We have conjectured that all consistent 6D supergravity theories with Lagrangian descriptions can be realized in string theory, and that this set of models can be identified from low-energy considerations.

In framing this conjecture of string universality in six dimensions, we hope we have provided a framework for concrete progress in understanding the 
string landscape and consistency conditions for low-energy field theories to be coupled to gravity. Progress in proving or disproving string universality in $6 \mathrm{D}$ theories, either through specific examples or general more abstract arguments will be of great value not only in six dimensions, but in developing our understanding of these questions for the more difficult but more important physical case of four dimensions.

\section{Acknowledgment}

We would like to thank Allan Adams, Michael Douglas, Eric Fitzgerald, David Guarrera, Ken Intriligator, Nabil Iqbal, John McGreevy, Greg Moore, David Morrison, Joe Polchinski, Vladimir Rosenhaus, Cumrun Vafa, and Edward Witten for discussions and correspondence related to this work. We are very grateful to Ilarion Melnikov for bringing to our attention a factor of two error in the original version of [29] that affected some statements in an earlier version of this paper. WT would like to thank the KITP for hospitality during the formative stages of this work. Thanks also to the organizers and participants of the conference "Perspectives on mathematics and physics," celebrating the 85th birthday of I. M. Singer and his legendary contributions to mathematics and physics, where some of this work was first presented, and particular thanks from WT also to I. M. Singer, for providing support and encouragement over many years as well as some of the inspiration for this work. This research was supported by the DOE under contract \#DE-FC02-94ER40818. This research was also supported in part by the National Science Foundation under Grant No. PHY05-51164.

\section{References}

[1] M. Grana, Flux compactifications in string theory: a comprehensive review, Phys. Rep. 423 (2006), 91, arXiv:hep-th/0509003; M.R. Douglas and S. Kachru, Flux compactification, Rev. Mod. Phys. 79 (2007), 733, arXiv:hep-th/0610102; F. Denef, M.R. Douglas and S. Kachru, Physics of string flux compactifications, Ann. Rev. Nucl. Part. Sci. 57 (2007), 119, arXiv:hep-th/0701050.

[2] O. DeWolfe, A. Giryavets, S. Kachru and W. Taylor, Type IIA moduli stabilization, J. High Energy Phys. 0507 (2005), 066, arXiv: hep-th/0505160.

[3] C. Vafa, The string landscape and the swampland, arXiv: hep-th/0509212.

[4] L. Alvarez-Gaume and E. Witten, Gravitational anomalies, Nucl. Phys. B 234 (1984), 269. 
[5] J.H. Schwarz, Anomaly-free supersymmetric models in six dimensions, Phys. Lett. B 371 (1996), 223, arXiv:hep-th/9512053.

[6] S.D. Avramis and A. Kehagias, A systematic search for anomaly-free supergravities in six dimensions, J. High Energy Phys. 0510 (2005), 052, arXiv:hep-th/0508172.

[7] M.B. Green, J.H. Schwarz and P.C. West Anomaly free chiral theories in six-dimensions, Nucl. Phys. B 254 (1985), 327.

[8] D.R. Morrison and C. Vafa, Compactifications of F-theory on Calabi - Yau threefolds-I, Nucl. Phys. B 473 (1996), 74, arXiv:hep-th/9602114; D.R. Morrison and C. Vafa, Compactifications of F-theory on Calabi - Yau threefolds-II, Nucl. Phys. B 476 (1996), 437, arXiv:hep-th/9603161.

[9] M. Bershadsky, K.A. Intriligator, S. Kachru, D.R. Morrison, V. Sadov and C. Vafa, Geometric singularities and enhanced gauge symmetries, Nucl. Phys. B 481 (1996), 215, arXiv: hep-th/9605200.

[10] P.S. Aspinwall, Point-like instantons and the spin(32)/Z(2) heterotic string, Nucl. Phys. B 496 (1997), 149, arXiv:hep-th/9612108; P.S. Aspinwall and D.R. Morrison, Point-like instantons on K3 orbifolds, Nucl. Phys. B 503 (1997), 533, arXiv:hep-th/9705104.

[11] M. Bershadsky and C. Vafa, Global anomalies and geometric engineering of critical theories in six dimensions, arXiv:hep-th/9703167.

[12] J.D. Blum and K.A. Intriligator, Consistency conditions for branes at orbifold singularities, Nucl. Phys. B 506 (1997), 223, arXiv:hep-th/9705030.

[13] J.D. Blum and K.A. Intriligator, New phases of string theory and 6D $R G$ fixed points via branes at orbifold singularities, Nucl. Phys. B 506 (1997), 199, arXiv:hep-th/9705044.

[14] R.G. Leigh and M. Rozali, Brane boxes, anomalies, bending and tadpoles, Phys. Rev. D 59 (1999), 026004, arXiv:hep-th/ 9807082.

[15] J. Park and A.M. Uranga, A note on superconformal $N=2$ theories and orientifolds, Nucl. Phys. B 542 (1999), 139, arXiv:hep-th/9808161.

[16] G. Aldazabal, D. Badagnani, L.E. Ibanez and A.M. Uranga, Tadpole versus anomaly cancellation in $D=4,6$ compact IIB orientifolds, J. High Energy Phys. 06 (1999), 031, arXiv:hep-th/9904071.

[17] M. Bianchi and J.F. Morales, Anomalies and tadpoles, J. High Energy Phys. 03 (2000), 030, arXiv:hep-th/0002149.

[18] A.M. Uranga, D-brane probes, $R R$ tadpole cancellation and $K$ theory charge, Nucl. Phys. B 598 (2001), 225, arXiv:hep-th/ 0011048. 
[19] A. Grassi and D.R. Morrison, Group representations and the Euler characteristic of elliptically fibered Calabi-Yau threefolds, arXiv:math/0005196.

[20] S. Kachru and C. Vafa, Exact results for $N=2$ compactifications of heterotic strings, Nucl. Phys. B 450 (1995), 69, arXiv: hep-th/9505105.

[21] C. Vafa, Evidence for F-theory, Nucl. Phys. B 469 (1996), 403, arXiv:hep-th/9602022.

[22] E.G. Gimon and J. Polchinski, Consistency conditions for orientifolds and D-manifolds, Phys. Rev. D 54 (1996), 1667, arXiv:hep-th/9601038; A. Dabholkar and J. Park, An orientifold of type-IIB theory on K3, Nucl. Phys. B 472 (1996), 207, arXiv:hep-th/9602030; R. Blumenhagen, V. Braun, B. Kors and D. Lust, Orientifolds of K3 and Calabi-Yau manifolds with intersecting D-branes, J. High Energy Phys. 07 (2002), 026, arXiv:hep-th/0206038.

[23] E.G. Gimon and C.V. Johnson, K3 Orientifolds, Nucl. Phys. B 477 (1996), 715, arXiv:hep-th/9604129; A. Dabholkar and J. Park, Strings on orientifolds, Nucl. Phys. B 477 (1996), 701, arXiv:hep-th/9604178; J. Polchinski, Tensors from K3 orientifolds, Phys. Rev. D 55 (1997), 6423, arXiv:hep-th/9606165.

[24] A. Salam and E. Sezgin, Anomaly freedom in chiral supergravities, Phys. Scr. 32 (1985), 283; E. Bergshoeff, T.W. Kephart, A. Salam and E. Sezgin, Global Anomalies in six-dimensions, Mod. Phys. Lett. A 1 (1986), 267; S.V. Ketov, Cancellation of gravitational anomalies in $D=6$ supergravities, Sov. Phys. J. 32 (1989), 801; S.V. Ketov, Anomalies of Kaluza-Klein theories in six-dimensions, Class. Quantum Grav. 7 (1990), 1387.

[25] H. Nishino and E. Sezgin, Matter and gauge couplings of $N=2$ supergravity in six-dimensions, Phys. Lett. B 144 (1984), 187; H. Nishino and E. Sezgin, The complete $N=2, D=6$ supergravity with matter and Yang-Mills couplings, Nucl. Phys. B 278 (1986), 353; H. Nishino and E. Sezgin, New couplings of six-dimensional supergravity, Nucl. Phys. B 505 (1997), 497, arXiv:hep-th/9703075; S. Ferrara, F. Riccioni and A. Sagnotti, Tensor and vector multiplets in six-dimensional supergravity, Nucl. Phys. B 519 (1998), 115, arXiv:hep-th/9711059; F. Riccioni, All couplings of minimal six-dimensional supergravity, Nucl. Phys. B 605 (2001), 245, arXiv: hep-th/0101074.

[26] M. Bianchi and A. Sagnotti, On the systematics of open string theories, Phys. Lett. B 247 (1990), 517; M. Bianchi and A. Sagnotti, Twist symmetry and open string Wilson lines, Nucl. Phys. B 361 (1991), 519.

[27] A. Sagnotti, A Note on the Green-Schwarz mechanism in open string theories, Phys. Lett. B 294 (1992), 196, arXiv:hep-th/9210127. 
[28] G. Honecker, Massive U(1)s and heterotic five-branes on K3, Nucl. Phys. B 748 (2006), 126, arXiv:hep-th/0602101; G. Honecker and M. Trapletti, Merging heterotic orbifolds and K3 compactifications with line bundles, J. High Energy Phys. 01 (2007), 051, arXiv:hep-th/0612030.

[29] V. Kumar and W. Taylor, Freedom and constraints in the K3 landscape, J. High Energy Phys. 05 (2009), 066, arXiv:hep-th/0903.0386.

[30] G. Aldazabal, S. Franco, L.E. Ibanez, R. Rabadan and A.M. Uranga, $D=4$ chiral string compactifications from intersecting branes, J. Math. Phys. 42 (2001), 3103, arXiv: hep-th/0011073.

[31] L.E. Ibanez, F. Marchesano and R. Rabadan, Getting just the standard model at intersecting branes, J. High Energy Phys. 0111 (2001), 002, arXiv: hep-th/0105155.

[32] M. Berkooz, R.G. Leigh, J. Polchinski, J.H. Schwarz, N. Seiberg and E. Witten, Anomalies, dualities, and topology of $D=6 N=1$ superstring vacua, Nucl. Phys. B 475 (1996), 115, arXiv:hep-th/9605184.

[33] J. Erler, Anomaly cancellation in six-dimensions, J. Math. Phys. 35 (1994), 1819, arXiv:hep-th/9304104.

[34] L.E. Ibanez and A.M. Uranga, $D=6, N=1$ string vacua and duality, in: "Seoul/Sokcho 1997, Dualities in gauge and string theories", 230282 [hep-th/9707075].

[35] E. Witten, Small instantons in string theory, Nucl. Phys. B 460 (1996), 541, arXiv:hep-th/9511030.

[36] M.J. Duff, R. Minasian and E. Witten, Evidence for heterotic/heterotic duality, Nucl. Phys. B 465 (1996), 413, arXiv:hep-th/9601036.

[37] A. Adams, N. Arkani-Hamed, S. Dubovsky, A. Nicolis and R. Rattazzi, Causality, analyticity and an IR obstruction to UV completion, J. High Energy Phys. 10 (2006), 014, arXiv:hep-th/0602178.

[38] N. Arkani-Hamed, H.C. Cheng, M.A. Luty and S. Mukohyama, Ghost condensation and a consistent infrared modification of gravity, J. High Energy Phys. 05 (2004), 074, arXiv:hep-th/0312099; N. Arkani-Hamed, P. Creminelli, S. Mukohyama and M. Zaldarriaga, Ghost inflation, J. Cosmol. Astropart. Phys. 04 (2004), 001, arXiv:hep-th/0312100.

[39] N. Seiberg and E. Witten, Comments on string dynamics in six dimensions, Nucl. Phys. B 471 (1996), 121, arXiv: hep-th/9603003.

[40] K.A. Intriligator, $R G$ fixed points in six dimensions via branes at orbifold singularities, Nucl. Phys. B 496 (1997), 177, arXiv:hep-th/9702038. 
[41] M.R. Douglas and W. Taylor, The landscape of intersecting brane models, J. High Energy Phys. 01 (2007), 031, arXiv:hep-th/0606109; V. Rosenhaus and W. Taylor, Diversity in the tail of the intersecting brane landscape, arXiv:hep-th/0905.1951.

[42] N. Arkani-Hamed, L. Motl, A. Nicolis and C. Vafa, The string landscape, black holes and gravity as the weakest force, J. High Energy Phys. 06 (2007), 060, arXiv:hep-th/0601001; H. Ooguri and C. Vafa, 'On the geometry of the string landscape and the swampland, Nucl. Phys. B 766 (2007), 21, arXiv:hep-th/0605264.

[43] M.R. Douglas and Z. Lu, Finiteness of volume of moduli spaces, arXiv: hep-th/0509224.

[44] A. Sen, F-theory and orientifolds, Nucl. Phys. B 475 (1996), 562, arXiv: hep-th/9605150.

[45] P.S. Aspinwall, K3 surfaces and string duality, in: Yau, S.T. (ed.) "Differential Geometry Inspired by String Theory", 1-95 [hep-th/9611137].

[46] M.B. Green and J.H. Schwarz, Anomaly cancellation in supersymmetric $D=10$ gauge theory and superstring theory, Phys. Lett. B 149 (1984), 117.

[47] B. Fiol, Populating the swampland: the case of $U(1)^{496}$ and $E_{8} \times$ $U(1)^{248}$, arXiv:hep-th/0809.1525.

[48] J. de Boer, R. Dijkgraaf, K. Hori, A. Keurentjes, J. Morgan, D.R. Morrison and S. Sethi, Triples, fluxes, and strings, Adv. Theor. Math. Phys. 4 (2002), 995, arXiv:hep-th/0103170.

[49] L. Susskind, The Anthropic landscape of string theory, in: Carr, Bernard (ed.) "Universe or Multiverse?", 247-266 [hep-th/0302219].

[50] M.R. Douglas, The statistics of string/M theory vacua, J. High Energy Phys. 05 (2003), 046, arXiv:hep-th/0303194. 
